
allemande

46-2 | 2014

Intellectuels et politique en Allemagne

\title{
Jürgen Fuchs : écrivain et homme d'action - une incarnation est-allemande de l'intellectuel « à la française »
}

\section{Sibylle Goepper}

\section{(2) OpenEdition}

Journals

Édition électronique

URL : https://journals.openedition.org/allemagne/1160

DOI : 10.4000/allemagne. 1160

ISSN : 2605-7913

Éditeur

Société d'études allemandes

\section{Édition imprimée}

Date de publication : 30 décembre 2014

Pagination : 393-408

ISSN : 0035-0974

\section{Référence électronique}

Sibylle Goepper, « Jürgen Fuchs : écrivain et homme d'action - une incarnation est-allemande de l'intellectuel « à la française » », Revue d'Allemagne et des pays de langue allemande [En ligne], 46-2 I 2014, mis en ligne le 29 juillet 2019, consulté le 29 mai 2021. URL : http://journals.openedition.org/ allemagne/1160; DOI : https://doi.org/10.4000/allemagne.1160 


\section{Jürgen Fuchs : écrivain et homme d'action - une incarnation est-allemande de l'intellectuel « à la française »}

\section{- Sibylle Goepper*}

Parmi les dissidents de RDA, Jürgen Fuchs (1950-1999) n'est pas le plus connu: lorsqu'il est question de voix critiques face au régime du SED, on cite plus volontiers Robert Havemann, Wolf Biermann ou encore Rudolf Bahro. Il a pourtant en commun avec tous de s'être opposé aux dérives de la république socialiste et d'y avoir fait l'objet de représailles, et avec les deux derniers d'avoir été contraint à quitter la RDA contre sa volonté, en l'occurrence en août 1977. Cette réception tient sans doute au fait que Fuchs est né dans les années 1950 et que si l'on s'en tient strictement à cette date, il fait partie, non pas de la "génération intermédiaire " (Ankunftsgeneration) à l'instar de Biermann et Bahro, mais de celle des «nés dedans » (Hineingeborene), dont les membres vont avoir de tout autres prémisses d'écriture (expérimentation formelle et jeu avec la langue), de même qu'un tout autre positionnement par rapport au socialisme (rejet de l'idéologie et de l'utopie) que ceux de leurs aînés. « De 1977 à 1980/81, Jürgen fut une star dans les milieux littéraires de RDA, avec Thomas Brasch, il fut une figure d'identification pour la nouvelle génération qui s'était émancipée du système ${ }^{(1)}$. Le rapprochement effectué ici par Lutz Rathenow est trompeur. Car, tandis qu'après son arrivée à l'Ouest, Thomas Brasch limite ses prises de position dans le champ politique ${ }^{(2)}$, Jürgen Fuchs s'implique fortement dans cette sphère. Si l'on considère le paysage intellectuel de la RFA de la période et, en son sein, celui des anciens de RDA, on peut aller jusqu'à dire que son dynamisme et son radicalisme sont uniques. À une époque où, devant l'échec du socialisme réel, les intellectuels se désengagent de l'espace public à l'Est aussi, son positionnement peut même paraître anachronique. Par ailleurs, Fuchs n'est pas qu'un intellectuel, au sens commun de spécialiste de la pensée. Si sa production possède une

\footnotetext{
* Maître de conférences à l'Université Jean Moulin Lyon 3.

1 Lutz Rathenow in: Udo Scheer, Jürgen Fuchs. Ein literarischer Weg in die Opposition, Berlin, Jaron, 2007, p. 216.

2 Voir l'article de Florence Baillet dans ce numéro.
} 
dimension conceptuelle et théorique, il est aussi un homme de terrain et d'action: malgré lui d'abord, lorsqu'il est incarcéré durant neuf mois à Hohenschönhausen, la prison du ministère de la Sûreté d'État (MfS) à Berlin, puis par choix, quand, depuis Berlin-Ouest, il fait circuler clandestinement des documents, des informations et du matériel de l'autre côté du Mur ou encore pratique son métier de psychologue auprès de populations défavorisées dans un centre d'écoute du quartier de Moabit.

Néà Reichenbach en Saxe, Fuchs développe son sens critique dans une famille d'ouvriers où l'on ironise lorsqu'il est question, conformément à ce que veut la doxa officielle, du « peuple au pouvoir » et en particulier au contact d'une grand-mère chrétienne qui, sans lui être hostile, ne s'en laisse pas conter par le nouveau régime ${ }^{(3)}$. La situation géographique du Vogtland a également son importance: les radios et télévisions de l'Ouest y sont captées et la proximité avec la République tchèque sensibilise la population à ce qui se passe du côté de Prague en $1968^{(4)}$. Soutenu par certains de ses enseignants, Fuchs fait ses débuts littéraires durant la période de dégel culturel qui suit l'arrivée d'Erich Honecker au pouvoir en 1971, dans le cadre de la promotion des jeunes poètes par les Jeunesses socialistes (FDJ). À cette époque, il fait toutefois aussi la connaissance d'aînés qui affichent leur distance face au socialisme réel. Hans Joachim Schädlich et Reiner Kunze participeront à la formation intellectuelle de celui qui s'est donné pour devise la phrase de Wolfgang Borchert: «Nous n'obéirons plus jamais aux coups de sifflet ${ }^{(5)}$.

L'événement le plus marquant de ces années est le passage par l'armée (NVA) de 1969 à 1971. Affecté à la télécommunication, ce qui lui laisse beaucoup de temps, Fuchs consigne tout ce qu'il voit et entend. Très vite, son écriture se tourne vers un réalisme du quotidien qui entend faire la lumière sur son environnement sans tabous. Les Gedächtnisprotokolle (« Procès-verbal d'un duel »), qui ne paraîtront pas à l'Est, mais chez Rowohlt en 1977, relatent les expériences traumatiques de l'enfance et de la jeunesse, tout en s'interrogeant sur la disposition de l'individu à fonctionner pour un système. C'est ce même questionnement qui pousse Fuchs à entamer des études de psychologie sociale à Iéna. "Apprendre pour mieux vivre ", tel est le mot d'ordre de la poignée d'étudiants qu'il fréquente à son retour de l'armée. Désireux de s'ouvrir au monde, ces jeunes gens commencent à afficher leur différence: vêtements, coupe de cheveux, musiques et lectures non-orthodoxes, jusqu'à la volonté déclarée de réformer le pays. Comme souvent en RDA, la « déviance » commence dans le domaine culturel pour peu à peu s'inscrire sur le terrain politique. En 1975, à l'aube de passer son examen de fin d'études, Fuchs est renvoyé pour " atteinte publique à la réputation de [son] université »; ses textes et poèmes sont trop critiques pour être tolérés. Par la suite, il est exclu de la FDJ et du SED auquel il avait adhéré en 1973 dans le but de faire " bouger les choses ».

Ce cas de sanction possède une valeur paradigmatique: les ennuis commencent dès lors que l'on se met à penser par soi-même et hors les clous. Parallèlement, la Stasi ouvre une procédure de surveillance (Operativer Vorgang) sous le nom de code « Pegasus ». Les «mesures de démoralisation » (Zersetzungsmaßnahmen) qui l'accompagnent, destinées à intimider et déstabiliser Fuchs ainsi que son entourage, se poursuivront à

3 De nombreuses informations nous ont été communiquées par Lilo Fuchs, la femme de Jürgen Fuchs, lors d'un entretien accordé le 8.3.2013 à Berlin.

4 Jürgen Serke, Das neue Exil. Die verbannten Dichter, Francfort-sur-le-Main, Fischer, 1985, p. 75-76.

5 Jürgen Fuchs, Pappkameraden, Reinbek, Rowohlt, 1981. C'est nous qui traduisons les citations. 
l'Ouest jusqu'à la chute du Mur, date à laquelle son dossier comptera 30 classeurs de 300 pages chacun ${ }^{(6)}$. En 1975, l'un des principaux reproches adressés à Fuchs par les autorités concerne ses contacts notoires avec Robert Havemann et Wolf Biermann. C'est au demeurant le soutien apporté au chansonnier déchu de sa nationalité en 1976 qui lui vaudra de connaître un sort particulièrement sévère comparé à celui d'autres auteurs ayant exprimé leur solidarité : arrêté par la Stasi, il est emprisonné à Hohenschönhausen de novembre 1976 à août 1977, puis expulsé à l'Ouest.

Le passage de RDA en RFA représente une profonde césure dans la biographie de Fuchs. À ce propos, il évoquera toujours « un refus en son for intérieur »; de même que la RFA restera à ses yeux une terre $d^{\prime}$ ' exil » ${ }^{(7)}$. C'est précisément la période de l'après-émigration qui nous occupera ici. Au cours de cette dernière, Fuchs va devoir trouver un équilibre entre sa volonté de témoigner sur la politique répressive pratiquée par le SED à l'égard de toute forme d'opposition d'un côté, et son refus d'être récupéré par ses adversaires politiques conservateurs en RFA de l'autre - car tout au long de son parcours critique, il restera ancré à gauche. Plus qu'anachronique, Fuchs incarne une figure d'intellectuel littéraire atypique dans le contexte allemand où, on le sait, la notion d'intellectuel engagé ou moderne « à la française » est difficilement transférable ${ }^{(8)}$ : émancipé de l'esprit partisan des premières années, renonçant à toute affiliation ou " encartage ", il reste néanmoins fortement impliqué dans les affaires politiques de son époque. Or, sur l'étroit sentier de l'indépendance et de l'engagement, il semble parvenir à trouver une voie entre intellectuel déconnecté du champ politique et intellectuel organique (Antonio Gramsci) ${ }^{(9)}$, et ainsi s'approcher de l'intellectuel autonome tel que l'a défini Pierre Bourdieu ${ }^{(10)}$.

\section{L'expulsion de RDA et l'arrivée à l'Ouest (1977-1983) : témoigner entre les lignes de fronts}

À son arrivée en RFA, le 3 septembre 1977, Jürgen Fuchs fait une déclaration de trois minutes à la télévision qui éclaire parfaitement sa position. Il précise d’abord n'avoir jamais voulu quitter la RDA pour la RFA - le terme qu'il emploie est celui d' « expulsion " (Abschiebung) - et n'avoir cédé que sous la menace d'une peine de dix ans de réclusion ${ }^{(11)}$. Il exprime ensuite sa solidarité et son souci pour d'autres dissidents:

6 Jürgen Fuchs, «Der Abschied von der Diktatur », in: Hans Joachim Sch ÄDlich (dir.), Aktenkundig, Berlin, Rowohlt, 1992, p. 21.

7 Jürgen Fuchs, " "Und dir möchte ich dringend raten, nicht weiter den Marxismus-Leninismus zu verdrehen" "(5 mars 1990), in: Frank Goyke, Andreas Sinakowski (dir.), Jetzt Wohin? Deutsche Literatur im deutschen Exil, Berlin, Unabhängige Verlagsbuchhandlung Ackerstraße, 1990, p. 33.

8 Voir l'article d'Anne Kwaschik dans ce numéro.

9 Sur le cas particulier des intellectuels en RDA, voir Werner MitTENZwEI, Die Intellektuellen. Literatur und Politik in Ostdeutschland 1945-2000, Leipzig, Faber \& Faber, 2001, p. 9-20.

10 Nous reprenons la définition de Pierre Bourdieu selon laquelle l'intellectuel est un être bi-dimensionnel réinvestissant dans l'espace public et le champ politique le capital symbolique acquis en respectant les valeurs d'autonomie de son champ d'origine. Pierre Bourdieu, « Die Intellektuellen und die Macht », Hambourg, VSA Verlag, 1991, p. 44.

11 Fuchs a certes signé une demande de sortie du territoire, mais suite à de telles pressions que l'absence de marge de manœuvre ne fait pas de doute. Jürgen FucHs, Vernehmungsprotokolle (1978), Berlin, Jaron, 2009. Noté VP dans la suite de cet article. 
Robert Havemann, en résidence surveillée depuis novembre 1976, et Rudolf Bahro, qui vient d'être arrêté après la parution d'un extrait de L'Alternative à l'Ouest. S'il dénonce clairement la situation des droits de l'homme en RDA, son ancrage politique est, lui aussi, sans ambiguïté. Fuchs insiste sur la nécessaire démocratisation du socialisme: « [Nous voulons] une société socialiste soucieuse de l'humain, progressiste, au sein de laquelle chaque individu peut respirer librement, pas un État policier qui espionne ses citoyens, les emprisonne, les expulse ou les chasse de leur pays " ${ }^{(12)}$. Ce discours, où perdure l'espoir de voir naître une alternative en RDA, le place très nettement du côté du socialisme réformiste ${ }^{(13)}$.

Il apparaît dès cette interview que la cible des attaques de Fuchs est, davantage que l'État est-allemand, la police politique du SED (Stasi). Cette instance, son fonctionnement et ses objectifs fournissent le fil rouge de ses réflexions et de son engagement au cours des années suivantes. Très rapidement, on assiste à la parution dans le Spiegel de la série "Du sollst zerbrechen » ${ }^{(14)}$ ("On va te faire craquer »), dans laquelle Fuchs étudie en précurseur la « psychologie noire " ( schwarze Psychologie » ${ }^{(15)}$ ) de la Stasi. Un an plus tard, sur le même modèle que l'ouvrage précédent, les Vernehmungsprotokolle ("Souvenirs d'interrogatoires »), conçus et appris par cœur en prison car Fuchs n'y avait pas la permission d'écrire, livrent un témoignage édifiant non seulement sur les méthodes employées pour faire plier le détenu, mais également sur les stratégies de survie mises en œuvre par ce dernier. Ainsi, l'« écriture simulée » ("simuliertes Schreiben », VP 20), qui consiste à faire semblant d'écrire sur le plateau de la table durant les interrogatoires, de même que le silence, opposé durant deux mois et demi aux agents, ainsi qu'au codétenu chargé de l'espionner dans sa cellule, visent à le soustraire à la pression ambiante et à déstabiliser ceux qui lui font face. Ce qui intéresse Fuchs en premier lieu, ce sont les dommages psychiques à long terme entraînés par ces expériences limite: "Le silence et la guerre de cellule sont des genres à risques " ${ }^{(16)}$, précisera-t-il dans son roman Magdalena (1998). Par l'écriture, Fuchs exorcise donc ses propres traumatismes, mais entend aussi soutenir et armer psychologiquement ceux qui ont vécu ou vivent l'équivalent de l'autre côté du Mur. Il sait en effet que la porosité de la frontière entre les deux États allemands permet à ses textes de circuler sous le manteau en RDA, notamment à Iéna. Il y est une référence pour une partie de la jeunesse qui va se rebeller toujours plus ostensiblement contre le régime ${ }^{(17)}$.

Les activités de Fuchs ne sont toutefois pas que livresques. Dès son arrivée, il cherche les contacts et les soutiens auprès d'anciens de la RDA (Heinz Brandt, Rudi Dutschke)

12 Fuchs est cité par U. SCHEen, Ein literarischer Weg (note 1), p. 183.

13 Dans sa préface, Wolf Biermann souligne que Fuchs est communiste. Jürgen FucHs, Gedächtnisprotokolle, Reinbek, Rohwohlt, 1977, p. 8. Noté GP dans la suite de cet article.

14 Jürgen Fuchs, « Du sollst zerbrechen », Der Spiegel, 43-47 (1977). La série est consultable en ligne.

15 Manfred Wilke, "Das Schutzkomitee Freiheit und Sozialismus », in: Ernest KuczYnski (dir.), Im Dialog mit der Wirklichkeit. Annäherungen an Leben und Werk von Jürgen Fuchs, Halle, Mitteldeutscher Verlag, 2014, p. 423.

16 Jürgen Fuchs, Magdalena. MfS, Memfisblues, Stasi, Die Firma, VEB Horch \& Gauck - ein Roman, Berlin, Rowohlt, 1998, p. 71.

17 À ce propos: Gerald Praschl, Roland Jahn. Ein Rebell als Behördenchef, Berlin, Ch. Links, 2011, p. 25-80. 
ou de personnalités dont il se sent proche humainement et moralement (Ralph Giordano, Manès Sperber). Il s'agit de façon très pragmatique de créer un réseau d'entraide pouvant être activé à tout moment afin de défendre la cause des droits de l'homme en RDA. À ceux qui lui conseillent de se consacrer à son œuvre, Fuchs déclare vouloir s'inscrire dans la mouvance de Heinrich Böll et du Günter Grass de l'époque: "C'est à cette tradition que nous appartenons. Pas à celle qui est refermée sur elle-même, assise à son bureau et coupée du monde " ${ }^{(18)}$. Fuchs s'implique entre autres dans le "Comité de soutien Liberté et Socialisme" (Schutzkomitee Freiheit und Sozialimus). Créé en 1976 sur une initiative de l'essayiste Hannes Schwenger, du rédacteur en chef du Spiegel Jörg R. Mettke, de l'historien et sociologue Manfred Wilke et de la journaliste Margret Frosch, le comité avait précisément eu pour première mission de le faire libérer. Placé sous le parrainage de personnalités en vue, venues pour la plupart du monde de la culture, telles que Heinrich Böll, Günter Grass, Friedrich Dürrenmatt, Hans Magnus Enzensberger ou encore Romy Schneider, son seul objectif est d'exercer une pression médiatique suffisamment importante sur le SED pour qu'il libère ses prisonniers politiques. De ce point de vue, il représente une initiative unique en son genre: sans être animé par l'anticommunisme de l'« Association des victimes du stalinisme » (Vereinigung der Opfer des Stalinismus), il rassemble des figures issues de la gauche ouest-allemande qui, pour la première fois, prennent publiquement position contre la politique du gouvernement est-allemand et se solidarisent avec des prisonniers en $\mathrm{RDA}^{(19)}$. Entre 1977 et 1979 , le comité $œ u v r e r a$ à la libération d'une quarantaine de citoyens.

Ces premières années à l'Ouest témoignent de la tension qui traverse l'existence de Fuchs. L'« exil » est l'occasion de faire la connaissance d'artistes et d'intellectuels qu'il n'aurait pas rencontrés s'il était resté en RDA, de découvrir et d'étudier des œuvres auxquelles il n'aurait pas eu accès (Freud, Adler, Fromm, Marcuse, Glucksmann). Fuchs est à l'époque surtout marqué par la pensée de Manès Sperber:

"Il n'est aucune vérité que nous dussions taire pour des raisons tactiques, aucune tromperie que nous fussions autorisés à passer sous silence, même pour un temps. C’est pourquoi le combat de la Gauche pour la liberté est toujours associé à une quête de vérité, qu'il aspire à davantage de connaissance et de conscience. La gauche authentique, contrairement aux représentants de la pseudo-gauche, n'a rien d'autre à proposer que ce combat, que cette recherche qui habituera l'homme à vivre en dehors et contre l'Absolu » ${ }^{(20)}$.

La lecture de ces lignes rédigées en 1953 est salvatrice pour l'homme de gauche que Fuchs demeure. Cette ouverture à des horizons intellectuels nouveaux représente la part « positive, agréable et enrichissante du séjour à l'Ouest » ${ }^{(21)}$. Cependant, Fuchs est également confronté à une solitude qui lui pèse. La médiatisation et l'activisme décrits plus haut ne doivent en effet pas occulter que la phase d'acclimatation à l'Ouest est longue et très difficile. Le sentiment d'étrangeté et la perte de repères, les difficultés

18 Cité in: U. Scheer, Ein literarischer Weg (note 1), p. 229.

19 Sur l'histoire de ce comité: M. Wilke, « Das Schutzkomitee » (note 15), p. 409-428.

20 Jürgen Fuchs, « “...Und wann kommt der Hammer?” Psychologie, Opposition und Staatssicherheit », Berlin, BasisDruck, 1990, p. 75.

21 Lettre citée in: U. ScheER, Ein literarischer Weg (note 1), p. 203. 
matérielles également, ne sont surmontés que petit à petit, entre autres avec l'ouverture au début des années 1980 du centre d'écoute de la Waldstraße à Moabit, un lieu de rencontre et de conseil qui lui permet de mettre à profit en tant que psychologue l'expérience de la répression politique et de l'expatriation, tout en la dépassant grâce à la confrontation à d'autres vécus.

L'isolement est toutefois essentiellement politique et idéologique. Or il va s'accroître dans les années 1980, lorsque la RFA s'engagera sur la voie de la détente et de la coopération vis-à-vis de la RDA. Après que le comité a cessé ses activités en 1979, il n'y a plus que peu de personnes pour suivre publiquement Fuchs sur la voie de la défense des droits de l'homme en Allemagne de l'Est. Au désintérêt des Allemands de l'Ouest s'ajoute l'incapacité des émigrés de RDA ("Übergesiedelte ») à se fédérer en un groupe qui serait visible dans l'espace public ${ }^{(22)}$ - la notion d'exil, que Fuchs utilise pour qualifier sa situation, fait du reste à elle seule largement débat parmi eux ${ }^{(23)}$. Mais plus encore que l'indifférence, l'Allemand de l'Est déclenche l'hostilité. La gauche traditionnelle ouest-allemande (que lui appelle « dogmatique ») en particulier le voit d'un mauvais œil exercer son droit d'intervention dans le débat public. Ce sera entre autres le cas en 1983, au sein de l'Union des écrivains de l'Ouest (VS), au moment où la loi martiale décrétée deux ans auparavant provoque l'interdiction de l'Union des écrivains et du PEN en Pologne. Non seulement Fuchs fustige l'absence de réaction de ses collègues, mais il entame des recherches afin d'établir le noyautage de cette institution par les communistes. Deux ans plus tard, il dénonce dans la presse le simulacre de discussions sur la paix entre auteurs de l'Est et de l'Ouest qui prévoient de faire l'impasse sur la question des droits de l'homme. L'article, qui rassemble un collectif d'auteurs, appelle à boycotter ces rencontres ${ }^{(24)}$. Ces épisodes sont l'illustration du rôle de "trouble-fête » (Störenfried) que Fuchs endosse à cette période dans le champ intellectuel ouest-allemand - rôle qui, nous le verrons, s'affirmera encore lors du tournant de 1989.

Fuchs n'hésite pas à regretter publiquement, dans les rares médias qui lui offrent une tribune (essentiellement la Frankfurter Rundschau), la pusillanimité de la gauche ouest-allemande vis-à-vis du SED, ainsi que la peur affichée du contact avec les dissidents et les opposants de RDA, qu'ils se trouvent à l'intérieur des frontières ou aient émigré ${ }^{(25)}$. À la déception ressentie devant l'accueil réservé par ceux en qui l'on pensait trouver des alliés s'ajoute la pression due au harcèlement ininterrompu par la Stasi pour qui Fuchs reste l'une des cibles principales. Même à l'Ouest - et ce sera encore plus vrai après le décès de Robert Havemann le 9 avril 1982 -, il fait figure d'ennemi d'État $n^{\circ} 1$ avec Wolf Biermann ${ }^{(26)}$. Ainsi Erich Mielke, chef de la Stasi, lance-t-il en

22 Sur ce point, voir Sibylle GOEPPER, «Les dialog-hefte: un détour par l'Ouest afin de réaffirmer quelques libertés fondamentales en RDA », in: Hélène Camarade et Sibylle Goepper (dir.), Résistance, dissidence et opposition en RDA, à paraître aux éditions du Septentrion en 2015. Pour exemple: Hans Joachim ScHÄDlich, «Ich bin nicht im Exil », Die Zeit, 30.3.1984.

24 « Mehrere Gründe, an der Berliner Begegnung nicht teilzunehmen », Frankfurter Rundschau, 22.4.1983.

25 Jürgen Fuchs, " Rede auf dem Schriftstellerkongreß in Mainz 11.3.'83 », tageszeitung, 23.3.1983.

26 Le 4 mars 1988, Anna Jonas, présidente du VS, adresse une lettre de protestation à l'organe du SED, le Neues Deutschland, qui avait accusé Fuchs d'être un agent des services secrets ouest-allemands. Le 14 mars 1988, la tageszeitung annonce la création par J. Fuchs, W. Biermann, Rainer Hirsch, Roland 
mai 1982 tour à tour un mandat d'arrêt et un avis de recherche contre lui, tous deux devant rester en vigueur jusqu'en $1999^{(27)}$.

\section{Le soutien aux groupes oppositionnels de RDA (1983-1989): agir de part et d'autre du Mur}

La surveillance étroite de la Stasi et les mesures de répression employées contre Fuchs s'expliquent par son activisme, mais également par son efficacité. Au cours des mois passés chez Robert Havemann en 1975 après son renvoi de l'université (28), l'homme a pris conscience de la nécessité de passer par les médias de RFA afin d'agir en RDA. Il a aussi beaucoup appris sur les méthodes concrètes de transit d'informations entre l'Est et l'Ouest. Même après son départ donc, le contact n'est jamais interrompu. Dédié dans un premier temps à maintenir le lien avec la famille et les amis en transmettant des nouvelles et des documents de manière aléatoire, le circuit utilisé subit une véritable évolution qualitative au fil du temps, puisque qu'il va servir à apporter, sous de multiples formes et de manière de plus en plus ciblée, un soutien idéel et matériel aux différents milieux oppositionnels sur le terrain. Il convient aussi de souligner d'emblée que ce circuit ne fonctionne pas à sens unique: les contacts de Fuchs à l'Est lui font également parvenir, entre autres par l'intermédiaire de personnes ayant des facilités à circuler sans contrôle, des informations qui lui permettent d'alimenter les médias ouest-allemands et de faire contrepoids à la politique médiatique officielle de la RDA.

Le pacifisme de pure façade de la république socialiste et la militarisation croissante de la société est-allemande sont des préoccupations centrales chez Fuchs. Tandis qu'il les thématise littérairement dans les romans Fassonschnitt (1984) et Das Ende einer Feigheit (1988), il consacre une partie de son énergie à soutenir le mouvement pacifiste indépendant (unabhängige Friedensbewegung) qui s'organise en RDA. En février 1982, il transmet à la Frankfurter Rundschau l'« Appel de Berlin. Pour une paix sans armes » (Berliner Appell. Frieden schaffen ohne Waffen) rédigé en janvier 1982 par Robert Havemann et Rainer Eppelmann, pasteur de la Samariterkirche ${ }^{(29)}$. Ce document, signé par plus de 80 militants pacifistes est-allemands, demande à ce que soient jetées les bases d'un ordre pacifique durable, qui ne se réduirait pas uniquement à l'absence de guerre. Notons en outre que l'on assiste là à un passage de relais entre générations, puisque, dans les mois qui suivent, Eppelmann deviendra le principal interlocuteur de Fuchs à Berlin-Est. Après la mort de Havemann, on assiste en effet à un changement de paradigme, y compris dans les modes de désobéissance face au pouvoir: le socialisme réformiste des anciens intellectuels marxistes cède la place à l'opposition à la fois plus directe et plus ciblée des comités de citoyens et des "groupes de base "(Basisgruppen).

Jahn, Freya Klier, Stephan Krawczyk, Rüdiger Rosenthal et les époux Templin d'un fonds d'aide aux victimes des calomnies du journal.

27 J. Fuchs, «Der Abschied» (note 6), p. 33.

28 Sur cette période: Andreas Sснміdт, «Unruhiges Refugium. Als Jürgen Fuchs bei Robert Havemann wohnte ", in: Bernd Florath (dir.), Annäherungen an Robert Havemann. Biographische Studien und Dokumente, Göttingen, Vandenhoeck \& Ruprecht. L'ouvrage paraîtra au printemps 2015.

29 Le document est consultable en ligne: http://www.jugendopposition.de/index.php?id=2157. Dernière consultation 17.08.2014. Voir également Erhardt Neubert, Geschichte der Opposition in der DDR 1949-1989, Bonn, Bundeszentrale für politische Bildung, 1997 (2 éd.), p. 408-409. 
Or Fuchs voit dans ces mouvements critiques envers le pouvoir, aspirant à davantage de démocratie, les représentants en RDA de la " gauche authentique » pensée par Sperber ${ }^{(30)}$.

À tous ses contacts, Fuchs demande des renseignements concrets sur la répression dont font l'objet les porteurs de symboles pacifistes lors de manifestations, les procès et les emprisonnements d'objecteurs de conscience. Il met ensuite en mouvement les relais médiatiques dont il dispose à l'Ouest afin d'obtenir leur libération. À Iéna, ce sont Bettina et Lutz Rathenow qui assurent le lien. Le 3 janvier 1983, alors que plusieurs militants pour la paix de Iéna sont dans les prisons de la Stasi suite à des actions menées dans le centre-ville, Fuchs adresse, par l'intermédiaire de la tageszeitung, une lettre ouverte au mouvement pour la paix européen afin qu'il apporte son soutien à son homologue de l'Est ${ }^{(31)}$. Devant la pression médiatique, le régime relâche les activistes avant l'heure. La plupart d'entre eux, parmi lesquels se trouve Roland Jahn, seront expulsés à l'Ouest quelques mois plus tard.

Fuchs est renseigné sur l'état d'esprit et les différentes actions des mouvements pour la paix est-allemands. Il en fait état en RFA et au niveau international, tandis qu'il répercute à l'Est des informations sur ce qui se passe à l'Ouest et la perception qu'on y a des événements se déroulant de l'autre côté du Mur. On aborde ici le deuxième aspect de son action. Étendre la visibilité, chercher la reconnaissance au-delà des frontières allemandes, à l'image de la déclaration de solidarité de Václav Havel en $1985^{(32)}$, permet non seulement de protéger les opposants face aux poursuites, mais également de stimuler les convergences entre groupes de l'Ouest et de l'Est - Berlin offrant pour ce faire une plateforme idéale -, afin de planifier des actions communes et d'accroître le poids des cercles oppositionnels de RDA. Là aussi cependant, des réticences se font sentir. Une fois de plus, c'est la connexion que Fuchs souhaite établir entre la question de la paix et celle des droits de l'homme qui dérange. L'objectif final dépasse en effet largement le cadre du combat pour infléchir la politique de l'OTAN en matière d'armement: il s'agit de discréditer les membres du SED en tant qu'interlocuteurs officiels, de réhabiliter la position des dissidents et enfin de mener à la démocratisation du socialisme de RDA. On ne s'étonne donc pas de voir apparaître des divergences. Noyautage des organisations de l'Ouest par les communistes, volonté de ne pas discréditer son propre mouvement en multipliant les revendications, une certaine perméabilité à la criminalisation par le SED des opposants de RDA dont on ignore tout, tels sont les facteurs qui expliquent que Fuchs, à l'instar des autres «Übergesiedelte " (émigrés), soit la plupart du temps tenu à l'écart des discussions menées à l'Ouest ${ }^{(33)}$.

Malgré les difficultés, des actions communes auront pourtant lieu grâce à l'entrée au Bundestag en 1983 de certains députés verts qui associent les deux dimensions dans leurs prises de position. Au cours de l'une d'entre elles, le 12 mai 1983, Petra

30 J. Fuchs, «...Und wann » (note 20), p. 73.

31 Lukas Bескмалn, "Jürgen Fuchs und die westdeutsche Friedensbewegung », in: E. KuCZYNSKI, Im Dialog (note 15), p. 450.

32 Václav Havel, "Anatomie einer Zurückhaltung. Dissidenten und westliche Friedensbewegung ", dialog-heft, 11 (1985) (voir note 35).

33 Sur les multiples freins au soutien du mouvement pour la paix est-allemand par la RFA, voir L. BECKMANN, « Jürgen Fuchs» (note 31), p. 441-453. 
Kelly, Gerd Bastian et Lukas Beckmann manifestent sur l'Alexanderplatz en arborant le slogan des objecteurs de conscience est-allemands "De l'épée au soc de charrue » («Schwerter zu Pflugscharen »). Après avoir été reçus par Erich Honecker, ils rencontrent Rainer Eppelmann, Bärbel Bohley et plusieurs militants pacifistes de province - avant d'être frappés quelques jours plus tard par une interdiction d'entrer sur le territoire est-allemand. Bien qu'un trait d'union soit tracé entre les intellectuels de gauche ouest-allemands et l'opposition à l'Est par l'intermédiaire de ces personnalités, les contacts restent restreints. Au sein du parti écologiste, on reproche à cette minorité de mettre en péril le dialogue RFA-RDA et les chances d'exercer une influence positive sur la politique du SED ${ }^{(34)}$.

Les bonnes relations avec certains membres des Verts et de la Liste alternative (AL) vont néanmoins permettre à Fuchs de développer un moyen de communication supplémentaire par le biais des dialog-hefte qui vont transiter vers l'Est à raison de plus d'un numéro par mois durant cinq ans, de 1985 à $1989^{(35)}$. La naissance de cette "revue de photocopies » (« Kopierzeitschrift») est rendue possible par des facteurs aussi bien matériels qu'humains: la situation stabilisée de Fuchs grâce à son travail de psychologue à Moabit, la rencontre avec Roland Jahn, expulsé de Iéna le 9 juin 1983, qui fait preuve de la même détermination à agir, le soutien actif de députés ouest-allemands de gauche qui écoutent ce que ces émigrés ont à dire sur la RDA, mettent à leur disposition des bureaux, des moyens de reproduction et de l'argent, et enfin la prise de risques de journalistes, de diplomates, de représentants de l'Église qui acceptent de transporter les numéros de l'autre côté du Mur.

Même si, au premier abord, les dialog-hefte ne sont qu'une simple revue de presse, ils présentent de multiples intérêts pour le chercheur: il s'agit d'abord d'une initiative unique en son genre à forte valeur symbolique. Ils permettent ensuite de retracer, grâce à l'analyse des informations transmises, la diversification des objectifs des cercles oppositionnels de RDA : on observe ainsi qu'en 1986, l'écologie et la défense des droits civiques sont devenues des revendications centrales. On constate pour finir que la sensibilisation des médias ouest-allemands est beaucoup plus importante que durant la première moitié de la décennie: la perquisition de la Bibliothèque de l'environnement (Umweltbibliothek) par la Stasi en 1987, puis les arrestations lors des manifestations dédiées à Rosa-Luxemburg et Karl-Liebknecht l'année suivante donnent lieu à une couverture sans précédent en RFA.

Si les dialog-hefte semblent prouver que l'« homme d'action » («Macher ») est, au moins en partie, parvenu à ses fins, ils apportent également un éclairage original sur le patrimoine intellectuel et culturel que l'auteur cherche à faire vivre en RDA après son départ. Ainsi, si plusieurs numéros reviennent en 1985 et 1986 sur les débats et controverses ayant eu lieu au sein des instances littéraires ouest-allemandes (VS et PEN), on trouve, à côté de cette actualité immédiate, de nombreux extraits d'ouvrages destinés

34 Thomas KleIN, «Frieden und Gerechtigkeit! ». Die Politisierung der Unabhängigen Friedensbewegung in Ost-Berlin während der 80er Jahre, Cologne, Böhlau, 2007, p. 178-179. Le dialog-heft, 13 (1986) (voir note 35) fait état d'un débat qui a eu lieu à ce sujet dans les nº 5 à 8 de la revue Kommune en 1986.

35 Les dialog-hefte rassemblent 71 numéros au total. Ils sont consultables à la Robert-Havemann-Gesellschaft de Berlin. Nous avons pu les étudier entre l'été 2012 et l'été 2013, grâce au soutien de Frank Ebert et de ses collaborateurs que nous remercions. 
à servir de «nourriture », mais également de « munitions intellectuelles " ${ }^{(36)}$ sur place. Tandis que des textes d'Ossip Mandelstam, Raymond Aron ou encore Albert Camus interrogent le rôle et les devoirs de l'écrivain et de l'intellectuel modernes, on trouve mentionnés ou reproduits, parfois même intégralement, des ouvrages qui contribuent à la réflexion sur l'histoire du $\mathrm{xx}^{\mathrm{e}}$ siècle: Die Unfähigkeit zu trauern de Alexander et Margarete Mitscherlich, Die zweite Schuld oder Von der Last ein Deutscher zu sein de Ralph Giordano, Essays zur täglichen Weltgeschichte de Manès Sperber, Ein deutscher Kommunist. Rückblicke und Perspektiven aus der Isolation de Robert Havemann, Logik der Rettung de Rudolf Bahro, Kommunismus und Sozialdemokratie: Eine Streitschrift de Peter Lübbe - entre autres. Mais ce ne sont pas uniquement les intérêts de l'homme de lettres qui s'expriment, le psychologue contribue à la réflexion sur la violence et la torture d'État en faisant transiter aussi bien des documents informatifs, comme les rapports d'Amnesty International ou des comptes rendus de congrès de spécialistes, que des textes plus théoriques (Bruno Bettelheim, Erziehung zum Überleben, Erich Fromm, Anatomie der menschlichen Destruktivität, Erwin Ringel, Zur Gesundung der österreichischen Seele).

Une autre fonction des cahiers consiste à continuer de faire vivre en RDA les auteurs émigrés ${ }^{(37)}$. Il est impossible de rendre compte de l'ensemble des documents transmis (interviews, entretiens, prises de position dans la presse, poèmes, extraits d'ouvrages inédits), mais quelques noms, toutes générations confondues, suffisent à rendre compte de l'étendue de la palette: Sascha Anderson, Rudolf Bahro, Jurek Becker, Horst Bienek, Wolf Biermann, Heinz Brandt, Thomas Brasch, Gabriele Eckart, Wolfgang Hilbig, Bernd Jentzsch, Sarah Kirsch, Uwe Kolbe, Günter Kunert, Reiner Kunze, Katja Lange-Müller, Erich Loest, Monika Maron, Frank Wolf Matthies, Herta Müller, Hans Noll, Helga M. Novack, Utz Rachowski, Ulrich Schacht, Hans Joachim Schädlich, Guntram Vesper, Richard Wagner, Gerhard Zwerenz. Au-delà de la RDA, l'intérêt de Fuchs pour le bloc de l'Est contribue à enrichir un peu plus le contenu des cahiers: parmi les personnalités représentées figurent les Russes Varlam Chalamov, Alexandre Soljenitsyne, Andrei Sakharow, Andreï Siniavski, Joseph Brodsky ou Anatoli Martchenko, l’Ukrainien Vassyl Stous, les Tchèques Petruška Šustrová, Ivan Martin Jirous, Jaroslav Seifert et Václav Havel, les Polonais Czesław Miłosz, Seweryn Blumsztajn, Adam Michnik et Jan Józef Lipski. Ces références hautement symboliques jettent de fait un pont entre littérature, exil, dissidence et opposition et possèdent un très fort potentiel d'identification. Avec elles, Fuchs transcende les frontières des États allemands. Il valorise un patrimoine idéel susceptible de créer un réseau et des synergies à grande échelle. Cette " orientation vers l'humain » ("humane Orientierung ") est, selon lui, le propre d'un authentique engagement à gauche.

Il n'en demeure pas moins que le centre de gravité des cahiers n'est plus le même avec le temps. La place moins importante réservée à la réflexion intellectuelle s'explique sans nul doute par l'accumulation d'événements politiques en 1987, 1988 et 1989. Ceux-ci retiennent toujours davantage l'attention des médias de l'Ouest, ce que Fuchs veut faire savoir aux opposants de RDA afin de les encourager dans leurs actions. On peut aussi supposer que la demande en termes d'information a changé: la dissidence

36 Entretien avec Lilo Fuchs (note 3).

37 Sur la réception des dialog-hefte en RDA, voir S. Goepper, «Les dialog-hefte» (note 22). 
animée par les intellectuels est-allemands venus des champs scientifiques et culturels (au contact desquels Fuchs s'est lui-même formé) a été supplantée par l'opposition de militants issus de la société civile qui ne pensent plus le système de manière globale, mais cherchent à agir sur lui dans des domaines précis.

Dans la biographie qu'il lui consacre, Udo Scheer souligne la progressive perte d'influence de Fuchs en RFA face à d'autres hommes d'action tels que Roland Jahn et Siegbert Schefke. Grâce aux appareils photos et aux caméras qu'ils font passer en fraude en RDA, ces derniers atteignent désormais avec leurs reportages télévisés un public beaucoup plus vaste ${ }^{(38)}$. À cela s'ajoute l'ascendant grandissant exercé par Sascha Anderson, Hineingeborener arrivé en 1986, qui, en élevant la léthargie et la passivité au rang de stratégies de résistance, impose dans l'espace public ouest-allemand un style d'insoumission face au pouvoir situé aux antipodes de celui de Fuchs. Ce dernier continue malgré tout à être considéré comme l'un des plus importants contacts des opposants de RDA. C'est donc en toute logique que, se présentant avec sa femme devant les membres du Neues Forum à l'automne 1989, il est accueilli sous les applaudissements.

\section{La décennie du «tournant» (1989-1999): faire la lumière sur le passé et pardonner}

Jürgen Fuchs n’a jamais accepté d'avoir été chassé de RDA. Le $1^{\text {er }}$ décembre 1989, il remet, en compagnie de Wolf Biermann et de Roland Jahn, un document demandant la réhabilitation de plus de 70 artistes est-allemands à Dietmar Keller, ministre de la Culture du gouvernement Modrow. Le lendemain, il s'exprime dans la presse:

«Elle existe la gauche authentique, cette opposition allemande qui ne s'est pas laissé corrompre [...]. Elle a vécu en résidence surveillée, a été interdite de publication, a subi les diffamations. C'est à elle que je pense. À ses mots sur le socialisme humain et démocratique, sur l'orientation vers l'humain qui a fini par remporter la victoire. Que d'années amères. Et quelle chance nous avons aujourd'hui " ${ }^{(39)}$.

L'isolement de Fuchs, y compris parmi les émigrés de RDA, a plusieurs fois été souligné dans cet article. En 1989 cependant, la fraction d'auteurs qui, une année plus tôt, avait déclaré la guerre au SED après l'arrestation et l'expulsion du chansonnier Stephan Krawczyk et de la dramaturge Freya $\mathrm{Klier}^{(40)}$, va se reformer pour ne plus se dissoudre durant près d'une décennie. Elle va notamment soutenir les militants pour les droits civiques dans leur combat pour l'ouverture des archives de la Stasi. Ainsi, tandis que début septembre 1990, Wolf Biermann participe à la deuxième occupation des locaux du MfS et à la grève de la faim organisée par Bärbel Bohley et Reinhard Schult entre autres, Fuchs s'occupe de sensibiliser l'opinion publique à leur principale revendication: mettre en sûreté 180 kilomètres d'archives ainsi que 16000 sacs de documents broyés.

38 Voir: Kontraste - Auf den Spuren einer Diktatur (DVD), Berlin, Bundeszentrale für politische Bildung, 2005 ( $2^{\mathrm{e}}$ éd.) et plus particulièrement la série « Aufbruch im Osten 1987-1989. Geheime Videos und mutige Bürger », $134 \mathrm{mn}$.

39 Jürgen Fuchs, « Der Stalinismus hat nicht gewonnen », tageszeitung, 2.12.1989.

40 Jürgen Fuchs, «Der Spielraum hat das Ausmaß einer Zelle », tageszeitung, 6.2.1988 et " Der Protest der Künstler: Ja es ist Krieg », Frankfurter Allgemeine Zeitung, 27.1.1988. 
L'ouvrage Aktenkundig, publié en 1992, fait apparaître dans son sommaire opposants de RDA et auteurs émigrés côte à côte. Cette belle unité doit cependant être relativisée car tous ne voient pas d'un très bon œil que les « anciens ", qui ont quitté le pays depuis parfois plus de dix ans, viennent se mêler de ce qui n'est plus forcément leurs affaires. La concurrence au sujet de l'incarnation de la "vraie » résistance, caractéristique des périodes post-dictatures, ne fait que commencer. Mais ce ne sont pas ces dissensions qui sont à l'origine de l'atmosphère polémique qui règne tout au long de la décennie 1990. Fuchs et ses alliés adoptent une attitude inflexible en matière de mise en lumière du passé qui fait peur et provoque la colère. Parce qu'ils refusent, entre autres, que soient distribués des "Persilscheine » (" certificats d'innocence ») avant que les coupables n'aient été identifiés et condamnés - " Pas d'amnistie avant d'avoir expié », tel est le mot d'ordre de l'époque -, ils se retrouvent à nouveau très isolés. Aux yeux de Fuchs cette position inconditionnelle est pourtant légitimée par la nature similaire des systèmes qui tombent en 1945 et 1989, une nature que révèle en particulier la langue utilisée par la Stasi dans ses rapports:

«Voilà à nouveau la langue que mon cas exige, avec toutes ses abréviations, la syntaxe qui l'accompagne, les appositions et les nominalisations, nous sommes en Allemagne, pas vrai, Victor Klemperer a écrit sa "LTI", à présent, c’est la "LQI" qui est écrite, la langue du quatrième Reich » ${ }^{(41)}$.

On imagine sans mal quelles réticences suscite le parallèle ainsi tracé entre Troisième Reich et socialisme réel de RDA. En établissant de telles connexions, Fuchs s'en prend non seulement de front au principal « mythe fondateur " de la république socialiste déchue, l'antifascisme, mais il interroge également sans ménagement les comportements de sa population - non sans s'inclure dans ce questionnement:

«Enfermer et exclure les individus, rejeter celui qui pense autrement ou dont l'apparence est différente, tout cela est profondément en nous, depuis 1933 au moins. Hoyerswerda et Hohenschönhausen, Auschwitz et Buchenwald, MfS et Gestapo sont en nous » ${ }^{(42)}$.

La volonté de faire la lumière sur les activités de la Stasi est vécue comme une inquisition, non seulement par une partie des intellectuels est-allemands, qui craint les accusations de compromission voire de collaboration, mais également par certains représentants de la gauche intellectuelle ouest-allemande qui ne peuvent plus considérer la RDA comme une alternative face à la République fédérale. Ils sont donc nombreux à reprocher à Fuchs de répandre, avec ses formules choc, un poison qui nuit à la réconciliation nationale. Ces reproches ne sont pas totalement infondés: la radicalité de la démarche de Fuchs apparaît par exemple lorsqu'il révèle, documents de la Stasi à l'appui en dépit du devoir de confidentialité s'appliquant à ces sources, la longue collaboration de Sascha Anderson avec le MfS, provoquant un scandale sans précédent, qui ternira à jamais l'étoile de la génération littéraire du Prenzlauer Berg ${ }^{(43)}$.

41 Jürgen Fuchs, « Landschaften der Lüge. Der “Operative Vorgang” Fuchs » (I), Der Spiegel, 47 (1991), p. 282.

42 Ibid., p. 287.

43 Jürgen Fuchs, «Landschaften der Lüge (II). Pegasus, Spinne, Qualle, Apostel », Der Spiegel, 48 (1991), p. $72-90$. 
Ce ne sont pourtant pas tant les cas individuels de compromission qui l'intéressent que le fonctionnement général du MfS. Dans la série «Landschaften der Lüge » ${ }^{(44)}$ parue dans le Spiegel à l'automne 1991, l'écrivain réalise un travail d'éclaircissement, qui n'est pas dépourvu de compassion envers les informateurs non officiels (IM), dont il dresse un portrait psychologique subtil. De plus, comme à son habitude, il a le souci d'éclairer par un travail théorique les mécanismes propres à la société est-allemande ( double langage » ou « duplicité sociale»), afin de transmettre des repères à la société. Après la «psychologie noire ", il étudie la "psychologie opérationnelle " "operative Psychologie " ${ }^{(45)}$ ), discipline au programme de l'École supérieure du MfS, et analyse en profondeur les méthodes de torture psychologique employées par ses agents. Tout au long de ce travail d'enquête et d'élucidation, Fuchs est convaincu de jeter les bases qui permettront aux Allemands de vivre ensemble à l'avenir. Or pour ce faire, il estime indispensable que les victimes obtiennent le respect. Celui-ci suppose, outre la réhabilitation, la connaissance et la reconnaissance du préjudice subi. Cette forme de réparation doit permettre aux anciennes cibles de reprendre leur vie en main: "Être une victime ne fait pas forcément de moi un homme meilleur. Je ne plaide du reste pas uniquement pour un adieu à la dictature, mais également pour un adieu au rôle de victime " ${ }^{(46)}$. C'est parce que ce processus ne peut faire l'impasse sur la vérité contenue dans les dossiers qu'il faut autoriser l'accès aux archives de la Stasi ${ }^{(47)}$.

Les réflexions de Fuchs dépassent à nouveau le plan de la psychologie individuelle lorsqu'il souligne par ailleurs l'intérêt historiographique du travail d'exhumation et d'exégèse des dossiers. Car s'il permet d'établir les responsabilités, il aide aussi $a$ contrario à montrer que tous les citoyens est-allemands n'étaient pas membres de la Stasi. À la reconnaissance de ceux qui n'ont pas cédé et qui en ont payé le prix s'ajoute la valeur d'exemple de l'opposition qui a réellement existé en RDA. Fuchs est enfin convaincu que la promotion d'une culture démocratique sur le long terme en Allemagne passe par ce deuxième travail sur le passé (zweite Vergangenheitsbewältigung) et qu'il doit être sans concessions. Si les spécialistes reconnaissent aujourd'hui que l'ouverture des archives de la Stasi a effectivement parfois eu pour conséquence que l'on surévalue leur contenu ${ }^{(48)}$, cette hypersensibilité face à la question de la violation des droits de l'homme et de la soumission à une dictature aura eu pour effet positif de permettre de régler rapidement et somme toute sainement cette douloureuse succession. Contrairement à ce que beaucoup pensaient, le combat mené par Fuchs et d'autres contre la tradition du « point final ( «Schlussstrich») n’a en rien déstabilisé la démocratie dans l’Allemagne réunifiée.

44 Jürgen Fuchs, «Landschaften der Lüge », Der Spiegel, 47-51 (1991). La série est consultable en ligne.

45 U. SCHEER, Ein literarischer Weg (note 1), p. 302-303.

46 Jürgen Fuchs, « Abschied von der Diktatur. Gespräch mit Jürgen Fuchs über Täter, Opfer und die Stasi-Akten ", Wochenpost, 12, 12.3.1992.

47 J. Fuchs, «...Und wann » (note 20), p. 41. Voir aussi: Jürgen Fuchs, « Keine Verwaltung des StasiErbes » (Stellungnahme zum Stasi-Akten-Gesetz im Berliner Reichstag), tageszeitung, 27.8.1991.

48 Sur ce point, lire la préface de l'ouvrage d'Ilko-Sascha Kowalczuk, Stasi Konkret. Überwachung und Repression in der DDR, Munich, C.H. Beck, 2013 - ici en particulier p. 9-11. 
Alors que pour de nombreux émigrés est-allemands une forme de normalité s'installe au fil des années passées en RFA, Jürgen Fuchs conserve intacte sa capacité à s'indigner face aux manquements du régime du SED. Il développe une forme d'hyperactivité qui se résume dans l'image du salon de son appartement de Tempelhof, transformé en agence de presse, où les piles de journaux sont si hautes qu'elles servent de siège aux invités. Bien qu'il déclare avoir connu la tentation de s'accommoder du système avant de rompre avec lui et ne pas être imperméable au doute, il y a chez lui quelque chose d'absolu et de radical qui dérange. D’ennemi d'État en RDA, il est devenu trouble-fête dans la RFA des années 1980. Après la chute du Mur, on lui accolera l'étiquette peu flatteuse d' " inquisiteur", et même celle, plus valorisante, de "juste » le sera toujours avec une ironie qui suggère le reproche. Le caractère sacrificiel de son action, l'intransigeance de son discours, son ton par moments moralisateur et outré n'ont pas non plus manqué de lui mettre à dos d'anciens compagnons de route ${ }^{(49)}$.

Contrairement à nombre d'intellectuels de gauche, Fuchs a toujours été convaincu que payer tribut au socialisme autoritaire de RDA ne servait en rien la cause de la gauche humaniste. Sa « douce clarté » (« sanfte Klarheit »), sa « douceur implacable " ( unerbittliche Sanftheit », GP 7) ont été mises à profit afin de servir la cause de l'humanisme et de la démocratie sans les déconnecter. C'est au nom de cet idéal que Fuchs n'hésite pas, parfois avec des mots très durs, à mettre ses collègues est- et ouest-allemands face à leurs responsabilités en $1989^{(50)}$, rappelant de manière forte que l'intellectuel se caractérise non seulement par son élan humaniste, mais également par son attitude critique envers tous les pouvoirs. Son ethos, qui consiste à « se mêler de ce qui le regarde " (Einmischung in eigene Angelegenheiten ${ }^{(51)}$ ), ne recouvre certes pas entièrement la figure de l'« intellectuel total » de Sartre, mais en faisant la preuve de son autonomie dans ses champs d'activité d'origine (la littérature et la psychologie), puis en passant à des préoccupations de plus en plus liées à l'histoire, la politique et l'universel, Fuchs opère bel et bien le glissement caractéristique de l'intellectuel engagé «à la française ». Cette trajectoire tient sans nul doute à la position particulière qu'il occupe dans le champ en tant qu'émigré: "Ceux qui vivent sur une frontière reposent toujours la question de la liberté, de la fuite, de la dictature, du pouvoir et du socialisme, libérant ainsi des angoisses, de l'espoir, des déceptions qu'ils récoltent ensuite " ${ }^{(52)}$. Le rejet par le champ d'origine (la RDA), associé au mauvais ajustement au champ d'arrivée (la RFA), a conduit Fuchs à une "seconde dissidence » ${ }^{(53)}$, cette notion désignant l'étape supplémentaire dans le processus d'autonomisation face au pouvoir que certains artistes de RDA émigrés en RFA ont franchie avant 1989 et qui les amène à un positionnement

49 À propos de la polémique très vive avec Günter Grass, voir Sibylle Goepper, « La convergence de la "seconde dissidence" de RDA et du "Centre des auteurs germanophones à l'étranger" lors de la réunification des clubs allemands du PEN: vers une gauche capable de faire son deuil », Revue d'Allemagne et des pays de langue allemande, 41/2 (2009), p. 297-309. Jürgen Fuchs, « Es gibt keine Koexistenz mit der Lüge », Die Welt, 24.9.1990. D'après le titre d'un de ses ouvrages paru en 1986 chez Rowohlt. J. FUCHs, «...Und wann » (note 20), p. 62. d'auteurs de RDA émigrés en RFA lors de la "querelle littéraire interallemande" des années 1990 ", thèse de doctorat, Université Lumière Lyon 2, 2008. 
particulièrement radical lors du «tournant ». La normalisation du rôle d'intellectuel dans les années 1990 pointée par Günther Rüther ${ }^{(54)}$ est, dans le cas présent, le résultat d'un itinéraire spécifique et d'un processus de maturation entamé dès les années 1970.

Cette inscription dans le champ politique a cependant un prix : en s'impliquant fortement dans les débats liés à la question allemande, Fuchs a mis sa carrière littéraire entre parenthèses. La conscience qu'il en a est très perceptible dans le recueil Tagesnotizen $^{(55)}$. S'il n'est guère étonnant dans le contexte extrêmement tendu des années 1990 que le roman Magdalena reçoive un mauvais accueil, force est de constater que sa littérature documentaire avait cessé d'intéresser la critique dès le milieu des années 1980. Ses détracteurs lui ont reproché de perpétuer la tradition de la « littérature victimaire "(Betroffenheitsliteratur). Mais il y a trop peu d'impuissance et de passivité chez celui qui " pour mieux résister, a décidé d’agir par lui-même " ${ }^{(56)}$, pour que ce jugement convainque. Les années 2000 ont vu réémerger, littérairement parlant, plusieurs écrivains issus de la génération des Hineingeborene. Fuchs n'en a pour sa part pas eu le temps. Atteint d'une maladie sanguine rare détectée en 1994, dont on ignore aujourd'hui encore l'origine exacte ${ }^{(57)}$, il décède en 1999, à l'âge de 48 ans. Auparavant, sa pensée et son action auront contribué à assurer la pérennité de la " gauche authentique », celle qui est capable de reconnaître ses erreurs et de faire son deuil ${ }^{(58)}$.

\section{Résumé}

Des années 1970 à la fin de la décennie 1990, Jürgen Fuchs (1950-1999) réalise un parcours d'intellectuel littéraire atypique entre la RDA et la RFA. Les textes critiques de cet ami de Wolf Biermann et de Robert Havemann lui valent d'être emprisonné et expulsé à l'Ouest en 1977, mais, tandis que de nombreux collègues ayant connu le même sort se détournent de la sphère politique, Fuchs s'y engage fortement. Ce psychologue de formation sera notamment l'un des premiers à témoigner sur les méthodes de la police politique est-allemande (Stasi). À l'heure où la gauche ouest-allemande privilégie la négociation avec le SED afin d'éviter un nouveau conflit mondial, Fuchs refuse de déconnecter paix et droits de l'homme, une attitude radicale qui lui vaut l'incompréhension de sa famille politique d'origine. Les cahiers de photocopies, qu'il fait parvenir clandestinement de l'autre côté du Mur de 1985 à 1989, proposent un tout autre dialogue: ils contribuent non seulement à alimenter les mouvements oppositionnels de RDA en informations touchant à leurs domaines de revendications, mais aussi à faire vivre en RDA la "gauche authentique " appelée de ses vœux en son temps par Manès Sperber. Lors du tournant de 1989, Fuchs militera, avec une fraction d'autres auteurs est-allemands, en faveur d'un bilan sans concessions sur la RDA. C'est ainsi que l'Allemand de l'Est, à mi-chemin

54 Günther Rüther, Literatur und Politik. Ein deutsches Verhängnis?, Göttingen, Wallstein, 2013, p. 9.

55 Jürgen Fuchs, Tagesnotizen, Reinbek, Rowohlt, 1978, p. 24.

56 J. Fuchs, « Abschied von der Diktatur» (note 46), p. 8-9.

57 U. SCHEER, Ein literarischer Weg (note 1), p. 361-373.

58 D’après Ralph Giondano, «Zur zweiten Verdrängung deutscher Geschichte », (Der Spiegel, 16.3.1992), europäische Ideen, 127 (2003), p. 13. 
entre homme d'action et utopiste, achèvera de se profiler comme une incarnation de l'intellectuel moderne "à la française ».

\begin{abstract}
From the 1970s to the late 1970s, Jürgen Fuchs (1950-1999) had a singular literary career between GDR and FRG. This friend of Wolf Biermann and Robert Havemann, wrote critical texts that led him to be imprisoned and exiled to West Germany in 1977. While the majority of his colleagues who shared the same fate turned their back on politics, Fuchs got very much involved in it. As a trained psychologist, Fuchs was one of the first writers to make people aware of the methods used by the East German political police, the Stasi. While in the 1980s, the West German Left tried to save peace by pursuing a policy of détente towards GDR, Fuchs always refused to disconnect peace from human rights. This radical stance isolated him from his political family. The photocopyreview he produced and smuggled into GDR from 1985 to 1989 served another purpose: not only they supported GDR Opposition by providing them with information, but they also preserved the spirit of the "Authentic Left" (Manès Sperber) in East Germany. During the Wende, Fuchs was one of the emigrant authors who spoke out for an uncompromising assessment of GDR. Both a man of action and a utopian, this East German writer appears as the epitome of the intellectual « à la française ».
\end{abstract}

\title{
Zusammenfassung
}

Der Weg des Schriftstellers Jürgen Fuchs (1950-1999) zwischen DDR und BRD ist einmalig. Wegen seiner Kritik am Realsozialismus wurde er 1977 wie viele andere DDRKünstler ausgebürgert. Im Gegensatz zu den meisten Übergesiedelten setzte sich aber der junge Freund von Robert Havemann und Wolf Biermann im politischen Feld stets weiter ein, unter anderem indem er sehr früh die Stasi-Methoden an die westdeutsche Öffentlichkeit brachte. Während die Westlinke in den 1980ern um des Friedens willen einen Entspannungskurs gegenüber der DDR einschlug, lehnte es Fuchs immer entschieden ab, Frieden und Menschenrechte auseinanderzuhalten. Daraus resultierte eine wachsende Isolation. Die von ihm erstellten „Kopierzeitschriften“, die er von 1985 bis 1989 von seinen Kontaktpersonen in die DDR hineinschmuggeln ließ, förderten einen völlig anderen Dialog: Sie unterstützten mittels gezielter Informationen die DDR-Oppositionellen und erhielten den Geist der "authentischen Linke“, wie Manès Sperber sie einmal vertrat, in der sozialistischen Republik am Leben. Zur Zeit der Wende machte sich Fuchs mit einigen anderen emigrierten DDR-Autoren für eine unabdingbare Aufarbeitung der DDR-Vergangenheit stark. So profilierte sich dieser Macher und Utopist definitiv als Intellektueller ,à la française“. 